\title{
Night-time sudden commencements observed by CHAMP and ground-based magnetometers and their relationship to solar wind parameters
}

\author{
H. Lühr ${ }^{1}$, K. Schlegel ${ }^{2}$, T. Araki ${ }^{3}$, M. Rother ${ }^{1}$, and M. Förster ${ }^{1}$ \\ ${ }^{1}$ Helmholtz Centre Potsdam - GFZ, German Research Centre for Geosciences, Potsdam, Germany \\ ${ }^{2}$ Max-Planck-Institute für Sonnensystemforschung, Katlenburg-Lindau, Germany \\ ${ }^{3}$ Polar Research Institute of China, Shanghai, China \\ Received: 15 December 2008 - Revised: 16 March 2009 - Accepted: 6 April 2009 - Published: 4 May 2009
}

\begin{abstract}
We have studied 41 Sudden Commencements (SC) using simultaneous magnetic field data from the CHAMP satellite and ground stations of the years 20002007. They are all night time events, since the influence of ionospheric currents on the SC is supposed to be minimal at night. This is confirmed by our study for geomagnetic latitudes below $\pm 40^{\circ}$. We further found that the onset times of the SC signature at satellite altitude and on the ground are the same within an uncertainty of $10 \mathrm{~s}$ and that the slopes of the corresponding magnetic field variation are very similar. For magnetic latitudes poleward of $\pm 40^{\circ}$ the amplitude of SCs increases both at the satellite and on ground, probably a consequence of field-aligned currents. CHAMP sometimes records small-scale magnetic variations different from the ground, which can be explained by local ionospheric currents. We also studied the relationship between the SC amplitude seen by CHAMP and the corresponding abrupt solar wind dynamic pressure change, using ACE data. Our results are compared with earlier studies using ground-based data and with theoretical expectations. It turns out that the induction effect in the Earth is quite small at low latitudes. Another important result is that the magnetic signature near the Earth is over-proportionally reduced for weak SC events. A discussion of accuracy and the uncertainty of our results completes the paper.
\end{abstract}

Keywords. Ionosphere (Electric fields and currents) - Magnetospheric physics (Solar wind-magnetosphere interactions; Storms and substorms)

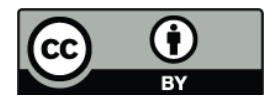

Correspondence to: $\mathrm{H}$. Lühr (hluehr@gfz-potsdam.de)

\section{Introduction}

It is generally accepted that the source of a sudden commencement (SC) is a sudden increase in solar wind dynamic pressure causing a compression of the magnetosphere. The resulting step in the magnetic field propagates earthwards as a hydromagnetic wave and can be observed as sudden increase mainly in the horizontal component of the magnetic field recorded for example by a ground-based magnetometer. With such ground-based magnetometers SCs have been studied for many years (e.g. Araki 1977, 1994; Petrinec et al., 1996; Engebretsen et al., 1999). Several workers have studied SCs observed at geosynchronous orbits and in the outer magnetosphere (e.g., Patel, 1972; Wilken et al., 1982; Kokubun, 1983; Cahill et al., 1990; Fowler and Russell, 2001). Recently SCs in the plasmasphere were studied by Shinbori et al. (2004).

Although SCs are known as a simple stepwise increase of the H-component, their amplitude and wave form show a complex global distribution depending upon local time and latitude. This is because secondary effects like field-aligned currents (FAC), ionospheric currents (IC) and tail currents (TC) contribute to the disturbance field of an SC in addition to the primary magnetopause current (MC). It becomes clear that the field-aligned currents may modify the SC-field even at low and middle latitudes (Kikuchi et al. 2001; Araki et al., 2006).

In order to understand the whole aspects of an SC it is important to separate the effects of each current source, MC, IC, FAC and TC. Among these currents IC plays an important role in causing the complex distribution of SC. The global distribution of the ionospheric current is deduced so far from ground-based observations, but it has to be confirmed by

Published by Copernicus Publications on behalf of the European Geosciences Union. 
simultaneous observations of low-altitude satellites above the ionosphere. Araki et al. (1984) analyzed 21 SC events observed by the Magsat satellite. They made a detailed case study for one $\mathrm{SC}$ in the evening sector at $50^{\circ}-60^{\circ}$ latitude. Both H- and D-components showed variations with opposite sense at the satellite and on ground. This was the first proof of ionospheric currents associated with SC. However, all the Magsat observations were from the dawn/dusk meridian due to the orbital configuration. This is a severe limitation for a general characterisation of SCs. In a recent study Han et al. (2007) made use of Ørsted magnetic field data for a direct comparison of satellite observations with ground-based recordings during $23 \mathrm{SC}$ events covering all local times. Han et al. (2007) reported that ionospheric currents do not contribute to the night side SCs by checking 15 nighttime SCs observed in the dip latitude range between $-35^{\circ}$ and $40^{\circ}$ and time sector 20:00-04:00 MLT. On the dayside they found clear differences between observations above and below the ionosphere reflecting the role of ionospheric currents.

Although the above studies have advanced our understanding of SCs, several issues are left open. Han et al. (2007) had in most cases to rely on ground-based data with 1 min resolution. This does not allow resolving the details of the SC temporal evolution. In case of the satellite observations they look only at the signature in the component aligned with the ambient field, which is indistinguishable from the variation in field strength. This can be considered appropriate at the magnetic equator, but causes already at $30^{\circ}$ invariant latitude an underestimation of the SC magnetic effect by about $30 \%$.

The time variation of the magnetopause current induces currents in the ionosphere and the Earth. The ionospheric currents generate horizontal magnetic fields with opposite signs above and below the ionosphere. While currents induced in the Earth cause magnetic fields in the same directions below and above the ionosphere. Simultaneous satellite and ground-based observations will therefore be useful in quantifying the role of these different currents. This issue could not be addressed by Han et al. (2007) because they interpreted only the variation in field strength at Ørsted.

Recognizing the questions raised above we make use of high-resolution vector magnetic field measurements by CHAMP, a low-altitude satellite and consider ground-based recordings at $1 \mathrm{~s}$ resolution. In this initial study we focus on the low and mid-latitude effects of SCs. Since we want to establish first the comparability of satellite and ground observations, we consider here measurements of $41 \mathrm{SC}$ events on the night side $(18: 00 \mathrm{~h}<\mathrm{MLT}<06: 00 \mathrm{~h})$. Due to the highly reduced conductivity, ionospheric currents should be negligible in this time sector. On the dayside, during sunlit conditions, quite different results are obtained. These will be discussed in a separate paper.

Our paper is organised as follows: in Sect. 2 we describe the satellite data as well as the simultaneous ground-based magnetometer data and their selection. In Sect. 3 we present typical examples of combined data from both sources and discuss the corresponding conclusions. Section 4 discusses effects of ground induction and ionospheric currents, and Sect. 5 contains the description of the solar wind data and their relationship to the observed SCs. In Sect. 6 we summarise and assess our results.

\section{Satellite and ground-based data and event selection}

The CHAMP satellite was launched in July 2000 and revolves the Earth on a circular and near polar (inclination $87.3^{\circ}$ ) orbit at an initial altitude of $450 \mathrm{~km}$ (in 2000) decaying to $350 \mathrm{~km}$ in 2007 (Reigber et al., 2002). The orbital plane precesses at a rate of $1 \mathrm{~h}$ in local time (LT) per 11 days, thus visiting all local times within 131 days.

The onboard Fluxgate Magnetometer (FGM) delivers vector field readings at a rate of $50 \mathrm{~Hz}$ and a resolution of $0.1 \mathrm{nT}$. The data are calibrated routinely with respect to the on- board absolute scalar Overhauser Magnetometer (OVM). This ensures a continuously high data quality. A dual-head star camera system mounted together with the FGM on an optical bench provides the orientation of the measured field vector with arc-second precision.

Magnetic field data used for this study are $1 \mathrm{~Hz}$ averages transformed first into a Mean-Field-Aligned (MFA) coordinate system. In this Cartesian local frame the z-axis is aligned with the mean field direction, the $y$-axis is perpendicular to the magnetic meridian, pointing eastward, and the $\mathrm{x}$ axis completes the triad. The magnetic field model POMME3 (Maus et al., 2006) has been used for defining the mean field direction.

In order to facilitate more easily the comparison with ground-based observations we performed an additional transformation into the commonly used components $H, D, Z$.

$H=B_{z} \cos I+B_{x} \sin I \quad D=B_{y} \quad Z=B_{z} \sin I+B_{x} \cos I$

where $I$ is the inclination of the geomagnetic field $\left(I=0^{\circ}\right.$ at equator, $I=90^{\circ}$ at North Pole). Since we are only interested in magnetic field variations, POMME-3 is also used for removing the main field.

Figure 1 shows for one example the $H, D, Z$ variations recorded by CHAMP during the SC event on 27 August 2001. The dashed vertical red line near 19:51 UT marks the reported onset time of the SC. Shortly after that the $H$ component starts to rise, reaching a peak deflection of about $40 \mathrm{nT}$ some $6 \mathrm{~min}$ later. The other components show little variations associated with the SC. This is remarkable since CHAMP moves more than $30^{\circ}$ in latitude during the event. From this example we can conclude that the chosen coordinate system is suitable for studying SCs from space and that the change in latitude during the event does not bias the measurements.

For the precise determination of the shock fronts in the solar wind stream we use a reliable and well-defined convection of the interplanetary magnetic field (IMF) and solar 
wind conditions to the magnetopause for any moment of the CHAMP observations. The IMF and solar wind data are taken from the Advanced Composition Explorer (ACE) spacecraft which is positioned near the L1 libration point between Sun and Earth. We have used data from the magnetic field instrument MAG (Smith et al., 1998) at 16-s resolution and the plasma instrument SWEPAM (McCormas et al., 1998) at 64-s resolution. MAG and SWEPAM data are resampled to one minute time resolution. Thereafter they are time shifted to represent solar wind and IMF conditions at the front side magnetopause, which is assumed to be located at a distance of $10 R_{E}$ in the $X_{\mathrm{GSE}}$ direction. We used the so-called phase front propagation technique (Weimer et al., 2003) in a slightly modified version that is based on a constrained minimum variance analysis of the IMF (Haaland et al., 2006). The orientation of the "phase fronts" is estimated from the IMF vector within two different time intervals around the actual ACE measurement. First it is applied to an approximately 8-min interval. If this fails, i.e., if the ratio between the eigenvalues is small $(<15)$, then a longer time interval of about $30 \mathrm{~min}$ is chosen and the analysis repeated. A relaxed lower threshold of 2 is used for the eigenvalue's ratio of the longer time interval. If neither interval gives a reliable normal, the previously valid orientation is kept. Missing ACE data are linearly interpolated if the gaps are shorter than $10 \mathrm{~min}$. Intervals with longer data gaps are not used at all. This concerns only a small fraction $(<1 \%)$ of the MAG data set and about $20 \%$ of the SWEPAM data.

In recent years many ground-based magnetometer stations have started to record the magnetic field with a time resolution of $1 \mathrm{~s}$ which is necessary to describe SC events in detail. Many earlier SC investigations were impeded by low time resolution. We used these 1-s resolution magnetic field data throughout our study whenever available.

The most versatile magnetometer network providing 1$\mathrm{s}$ resolution data is the Circum-Pan-Pacific-MagnetometerNetwork (CPMN) operated by the Kyushu University, Japan (e.g. stations ANC, DAW, EUS, HER, LAQ, SMA, TRD in the following figures). We also used data from the Australian Network operated by IPS Radio and Space Services (CAN, DAV), the MEASURE network of the Institute of Geophysics and Planetary Physics, UCLA (JAX, APL) and the Memambetsu and Kakioka Magnetic Observatories, Japan (MMB, KAK).

Between its launch and end of 2007 CHAMP sampled in total 201 SC events. For this study we selected events having occurred when CHAMP was on the night side (between 18:00 and 06:00 MLT), as already mentioned. In addition, we restricted our analysis to events observed at mid to low latitudes $\left(-50^{\circ}<\mathrm{MLat}<50^{\circ}\right)$ in order to avoid contamination from auroral currents enhanced by night-time particle precipitation. These two restrictions left us with $45 \mathrm{SC}$ events from which 4 could not be used because of gaps in the CHAMP records. Details of the remaining 41 cases are summarised in Table 1.

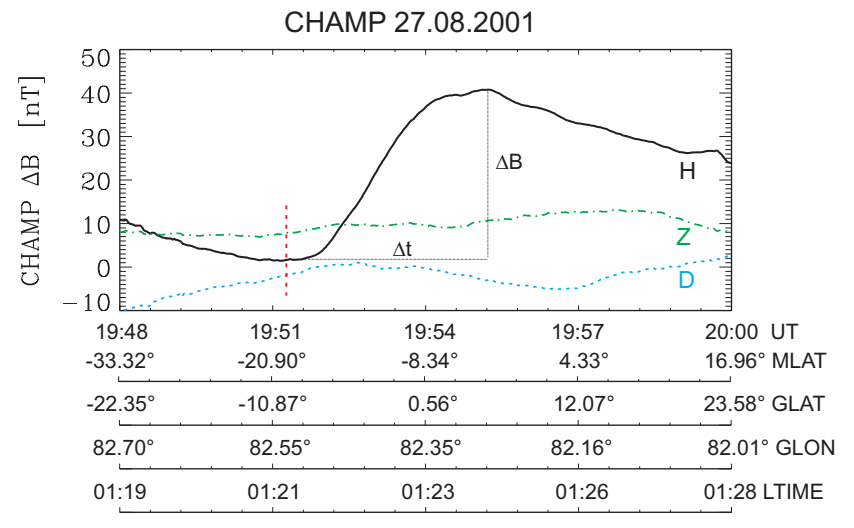

Fig. 1. Example of an SC recorded by the CHAMP magnetometer. $H, D, Z$ are the three components measured onboard CHAMP. The dashed red line marks the onset of the SC, $\Delta B$ is its amplitude (listed in Table 1 for all events).

\section{Examples of SC events}

From our sample of 41 night side events we present here four SCs in more detail occurring at different local times and locations on the globe (Figs. 2-5).

In each of these figures we plotted the magnetic variations of the $H$ component, as measured by CHAMP, together with the corresponding variations at four ground stations as close as possible to the CHAMP trajectory. The CHAMP and the four ground-based magnetic variations have been plotted on the same time axis. Magnetic field baseline values are subtracted such that the five curves all cross zero at the time of the SC onset (marked by a dashed vertical line). The locations are displayed in the right-hand part of the figures. Here the dashed line indicates the CHAMP orbit and the flight direction (arrow head). The data plotted in the left panel are from the orbit arc drawn as solid line, starting at the time of the SC (asterisk).

\subsection{November $2002 \mathrm{SC}$}

During the event shown in Fig. 2 CHAMP samples the time sector before midnight. The amplitude and waveform of the CHAMP magnetic variations are very similar to that at the stations DAW $\left(\right.$ MLat $\left.=-22^{\circ}\right)$, DAV $\left(-2^{\circ}\right)$ and MMB $\left(35^{\circ}\right)$ at nearly the same local time. CAN $\left(-43^{\circ}\right)$ shows a larger amplitude than the other stations. CHAMP recordings deviate, however, significantly from the ground observations at elapse times past $500 \mathrm{~s}$. This is surprising since CHAMP passes overhead of DAW at that time. We will address this apparent inconsistency in Sect. 4.

\subsection{November $2000 \mathrm{SC}$}

In this case (cf. Fig. 3) measurements are taken after midnight. Here again deflections in the $H$ component of CHAMP fit well the recordings at the near-by observatories. 
Table 1. List of all night-time SC events studied in this paper. Columns 2 to 4 give the date and the time when CHAMP encountered the SC event, columns 5 to 9 the geographic and geomagnetic locations at the encounter (Dir states the motion of CHAMP, polewards or equatorwards). Column 10 denotes the amplitude of the SC in the H-component (see Fig. 1), Colum 11 contains an estimate of the rise time of the SC from CHAMP data, Colums 12 to 14 the solar wind parameters derived from ACE data, and the last column the 3-hr $a_{p}$ value when the event occurred. For events no. 5, 13, 17 no solar wind data are available, in events no. 14, 26, 28, 41 no clear peak was observed in the CHAMP data, the horizontal component rose from the onset of the SC almost continuously towards high latitudes.

\begin{tabular}{|c|c|c|c|c|c|c|c|c|c|c|c|c|c|c|}
\hline no & Date & $\mathrm{UT}[\mathrm{h}]$ & $\mathrm{LT}[\mathrm{h}]$ & GLat $\left({ }^{\circ}\right)$ & Dir & GLon $\left(^{\circ}\right)$ & $\operatorname{MLT}[\mathrm{h}]$ & MLat $\left({ }^{\circ}\right)$ & $\Delta B[\mathrm{nT}]$ & $\Delta B / \Delta t[\mathrm{nT} / \mathrm{min}]$ & $\Delta p[\mathrm{nPa}]$ & $p_{1}[\mathrm{nPa}]$ & $B_{z}[\mathrm{nT}]$ & ap [nT] \\
\hline 1 & 280700 & 6.558 & 01.38 & -26.2 & pw & -77.7 & 01.32 & -13.4 & 46 & 9.0 & 4.5 & 0.9 & -7.7 & 39 \\
\hline 2 & 100800 & 5.005 & 00.17 & -15.0 & pw & -72.6 & 00.10 & -2.5 & 12 & 1.6 & 2.4 & 0.9 & -1.3 & 12 \\
\hline 3 & 061100 & 9.794 & 03.98 & -38.7 & eqw & -87.2 & 04.41 & -26.2 & 8 & 9.1 & 2.2 & 0.9 & -5.4 & 48 \\
\hline 4 & 281100 & 5.511 & 02.09 & -15.8 & eqw & -51.3 & 02.20 & -8.6 & 20 & 12.7 & 5.0 & 2.5 & -1.8 & 48 \\
\hline 5 & 220301 & 13.683 & 03.62 & 37.8 & eqw & -150.8 & 02.96 & 37.7 & 17 & 4.8 & - & - & 6.4 & 18 \\
\hline 6 & 030801 & 7.254 & 03.70 & 22.5 & pw & -53.4 & 04.01 & 27.9 & 28 & 5.3 & 6.9 & 2.1 & -3.0 & 32 \\
\hline 7 & 120801 & 11.576 & 02.62 & -41.7 & eqw & -134.3 & 03.00 & -37.6 & 29 & 6.5 & 7.0 & 1.5 & 4.3 & 22 \\
\hline 8 & 270801 & 19.858 & 01.36 & -9.1 & eqw & 82.5 & 01.43 & -19.0 & 40 & 10.9 & 7.5 & 2.2 & -0.9 & 39 \\
\hline 9 & 251001 & 8.810 & 20.09 & 6.4 & pw & 169.1 & 20.08 & 1.6 & 39 & 5.7 & 5.2 & 1.1 & 1.0 & 9 \\
\hline 10 & 281001 & 3.308 & 19.65 & -41.2 & eqw & -114.8 & 20.34 & -33.4 & 41 & 15.4 & 4.5 & 0.7 & -5.5 & 111 \\
\hline 11 & 291201 & 5.626 & 01.99 & 43.1 & eqw & -54.6 & 03.03 & 49.4 & 34 & 8.9 & 14.5 & 3.5 & 4.9 & 27 \\
\hline 12 & 230302 & 11.600 & 18.56 & -24.7 & pw & 104.3 & 18.39 & -36.3 & 15 & 3.0 & 3.8 & 1.0 & -0.7 & 15 \\
\hline 13 & 290302 & 22.608 & 05.76 & -34.3 & eqw & 107.2 & 05.18 & -46.9 & 29 & 4.6 & - & - & 5.1 & 18 \\
\hline 14 & 140402 & 12.566 & 04.51 & 15.6 & pw & -120.9 & 04.14 & 20.8 & - & - & 1.8 & 3.0 & -21 & 32 \\
\hline 15 & 230402 & 4.803 & 03.57 & -27.1 & eqw & -18.5 & 02.84 & -31.0 & 44 & 14.3 & 7.4 & 1.6 & 2.5 & 80 \\
\hline 16 & 200502 & 3.662 & 01.35 & 39.0 & pw & -34.8 & 01.96 & 39.0 & 33 & 9.2 & 3.1 & 1.6 & 5.3 & 12 \\
\hline 17 & 080602 & 11.660 & 23.38 & -17.7 & eqw & 175.8 & 23.47 & -22.3 & 24 & 7.7 & - & - & 2.5 & 7 \\
\hline 18 & 250702 & 13.610 & 19.08 & -18.8 & eqw & 82.0 & 18.64 & -29.5 & 3 & 2.3 & 0.8 & 1.2 & 5.7 & 12 \\
\hline 19 & 010802 & 23.159 & 18.09 & -63.9 & eqw & -76.0 & 18.76 & -49.4 & 24 & 5.9 & 1.9 & 0.4 & 3.5 & 39 \\
\hline 20 & 260802 & 11.510 & 04.31 & -25.0 & pw & -108.0 & 04.19 & -17.3 & 27 & 4.8 & 3.7 & 3.2 & -2.1 & 18 \\
\hline 21 & 091102 & 18.825 & 21.29 & 17.4 & eqw & 37.0 & 21.51 & 9.9 & 15 & 4.5 & 3.5 & 3.1 & 6.0 & 12 \\
\hline 22 & 111102 & 12.505 & 21.17 & 6.6 & pw & 130.0 & 21.31 & -1.4 & 22 & 10.5 & 4.0 & 4.0 & 4.5 & 22 \\
\hline 23 & 221202 & 10.470 & 05.33 & -33.5 & eqw & -77.1 & 05.88 & -20.5 & 9 & 1.8 & 1.4 & 1.2 & 3.2 & 7 \\
\hline 24 & 201103 & 8.045 & 22.83 & -44.6 & eqw & -138.2 & 23.97 & -41.2 & 66 & 24.4 & 11.8 & 2.2 & -3.9 & 94 \\
\hline 25 & 220104 & 1.606 & 05.05 & 48.8 & eqw & 51.7 & 05.02 & 44.4 & 38 & 14.9 & 14.0 & 2.3 & 1.3 & 48 \\
\hline 26 & 030404 & 14.148 & 22.60 & 3.7 & pw & 126.8 & 22.42 & -4.4 & - & - & 5.2 & 1.9 & -3.0 & 39 \\
\hline 27 & 090404 & 2.550 & 22.38 & -55.8 & pw & -62.6 & 21.98 & -41.9 & 32 & 4.6 & 3.7 & 1.7 & -3.5 & 18 \\
\hline 28 & 220704 & 10.600 & 00.72 & 45.9 & pw & -148.2 & 23.75 & 46.0 & - & - & 3.6 & 0.7 & -1.8 & 12 \\
\hline 29 & 300704 & 21.210 & 23.94 & 44.4 & pw & 40.9 & 00.31 & 39.7 & 26 & 1.9 & 2.4 & 0.8 & 0.1 & 18 \\
\hline 30 & 290804 & 10.082 & 21.06 & 5.6 & pw & 164.7 & 20.70 & -0.1 & 19 & 4.4 & 2.1 & 1.3 & 2.8 & 6 \\
\hline 31 & 130904 & 20.033 & 19.80 & 43.4 & pw & -3.5 & 20.27 & 38.6 & 56 & 19.4 & 10.0 & 2.0 & -0.4 & 32 \\
\hline 32 & 220904 & 6.564 & 19.60 & 47.4 & pw & -172.7 & 18.38 & 43.3 & 23 & 8.2 & 3.4 & 1.6 & 1.1 & 12 \\
\hline 33 & 271004 & 12.188 & 03.55 & 20.6 & eqw & -129.6 & 03.27 & 24.5 & 10 & 1.6 & 1.3 & 1.6 & 0.4 & 6 \\
\hline 34 & 111104 & 17.166 & 02.19 & 7.3 & pw & 135.5 & 02.33 & -0.6 & 27 & 4.3 & 4.8 & 2.5 & 2.0 & 15 \\
\hline 35 & 150505 & 2.640 & 21.16 & -28.6 & eqw & -82.2 & 21.20 & -16.2 & 75 & 33.8 & 20.5 & 2.0 & 0.2 & 67 \\
\hline 36 & 170705 & 01.575 & 03.21 & 54.4 & eqw & 24.4 & 03.69 & 50.4 & 15 & 2.3 & 2.4 & 2.7 & 3.0 & 12 \\
\hline 37 & 110905 & 12.735 & 22.20 & 18.0 & eqw & 142.0 & 21.83 & 10.5 & 43 & 6.2 & 5.8 & 1.6 & -3.8 & 132 \\
\hline 38 & 270706 & 13.888 & 04.59 & -53.4 & eqw & -139.5 & 05.57 & -49.5 & 6 & 1.8 & 1.6 & 3.0 & -1.7 & 12 \\
\hline 39 & 141206 & 14.233 & 03.86 & 22.2 & eqw & -155.8 & 03.91 & 22.2 & 50 & 8.1 & 7.5 & 1.3 & -0.3 & 48 \\
\hline 40 & 070507 & 08.430 & 02.37 & -57.0 & eqw & -91.0 & 02.93 & -44.0 & 18 & 2.2 & 3.6 & 6.0 & -4.7 & 15 \\
\hline 41 & 251007 & 11.600 & 22.62 & 50.8 & eqw & 165.3 & 22.31 & 44.6 & - & - & 1.8 & 6.2 & -3.2 & 15 \\
\hline
\end{tabular}

The station JAX, located at somewhat higher magnetic latitude $\left(41.8^{\circ}\right)$, observes a stronger SC signal.

\subsection{April 2002 SC}

Observations, made shortly before dawn, are presented for this event (cf. Fig. 4). CHAMP samples the SC on its way from mid-latitude towards the equator. The obtained magnetic signatures are quite similar to the recordings at EUS and VSS ( $1 \mathrm{~h}$ in LT earlier). At HER $\left(-34^{\circ}\right)$, located in the dawn sector, the SC response is significantly smaller. Conversely, the higher latitude station APL $\left(50^{\circ}\right)$ exhibits a much larger excursion. Interestingly, at this station a so-called preliminary reverse impulse (PRI) occurs (Araki et al., 1984). A PRI is a common feature of an SC signature on the dayside, but it is not expected at 02:00 LT.

\subsection{April 2004}

This final example, shown in Fig. 5, compares satellite and ground-based recordings from a somewhat different latitude range. Here CHAMP is heading towards higher latitudes over the course of the SC event. From the comparison between ground stations we can see that the step in field 


\section{Nov. 2002}
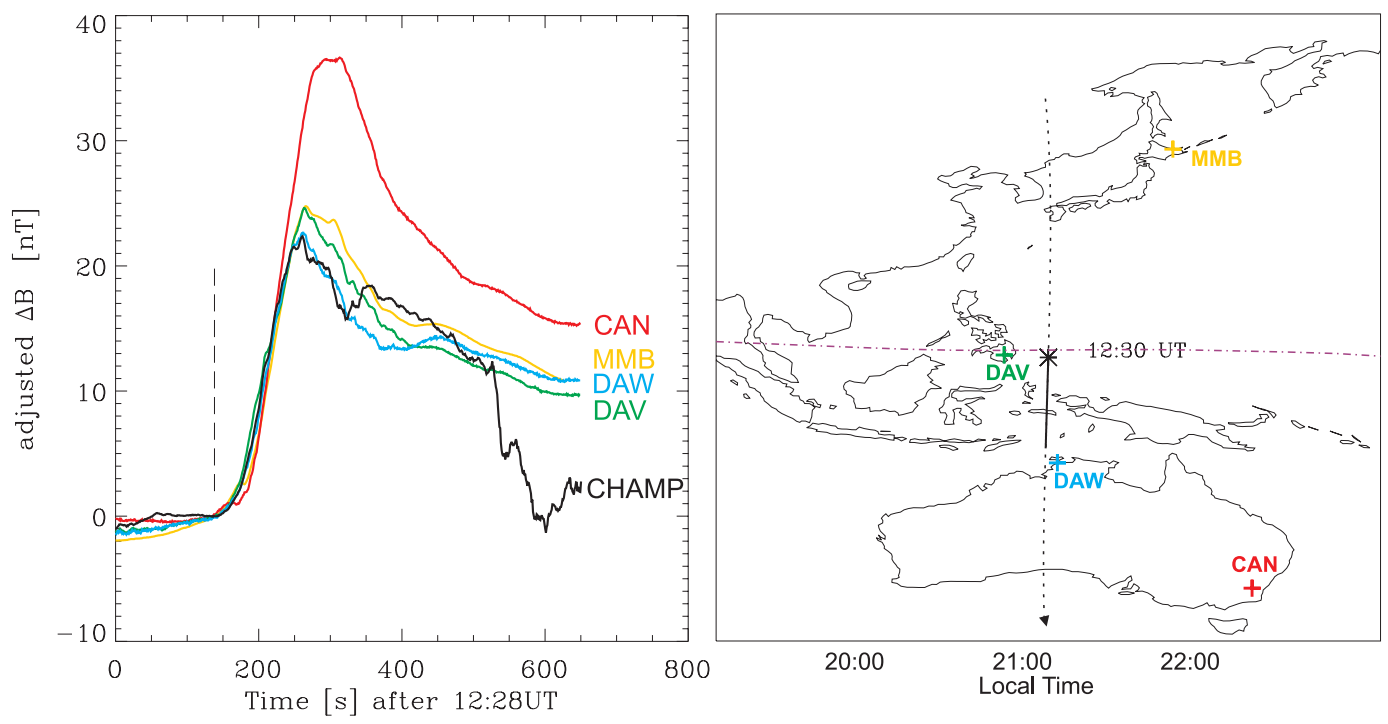

Fig. 2. The SC event of 11 November 2002 recorded by CHAMP (black) and at four ground stations (coloured) in the western Pacific region. The CHAMP trajectory and the locations of the ground stations are indicated on the map at the right hand side. The asterisk on the CHAMP track marks the position at the time of the SC onset. The dashed-dotted line reflects the geomagnetic equator.

\section{Nov. 2000}
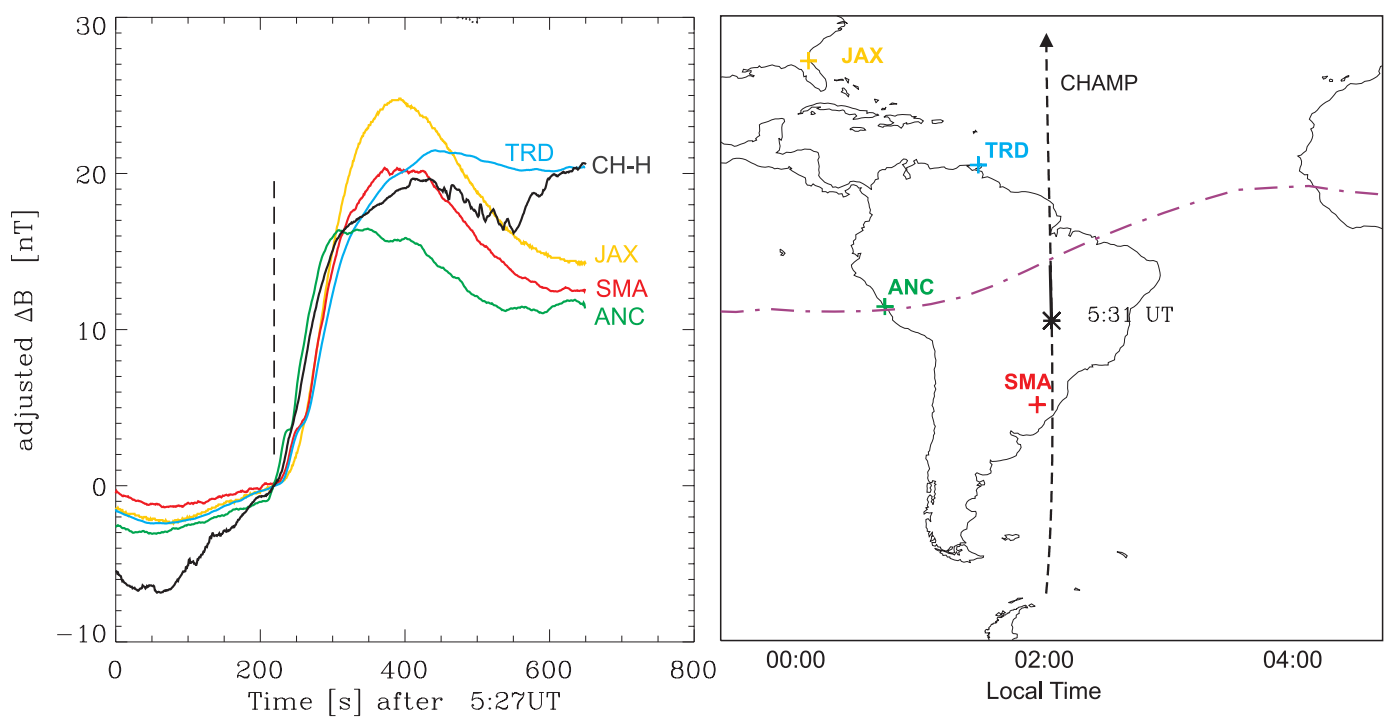

Fig. 3. Same as Fig. 2, but for the SC event on 2 November 2000, when CHAMP flew over South America.

increase gets larger towards higher magnetic latitudes. The curve of the CHAMP $H$ component lies between the CAN $\left(-43^{\circ}\right)$ recordings and the measurements at the lower latitude stations. When CHAMP passes the latitude of CAN the two signal curves are crossing. This indicates that the magnetic deflection caused by an SC continues to rise towards higher latitudes. Current systems responsible for that are discussed in Sect. 4.
The salient points derived from the four examples can be summarised as follows (these statements are also valid for the remaining $37 \mathrm{SC}$ events, not shown here):

- The onset time of the SC detected by CHAMP and the four ground-based stations is the same with a spread of the order of $10 \mathrm{~s}$. From our 1-s sampled data it can be judged that a latitudinal dependence (within $\pm 50^{\circ}$ MLat) of the SC onset time does not exist or is less than $10 \mathrm{~s}$. 


\section{April 2002}
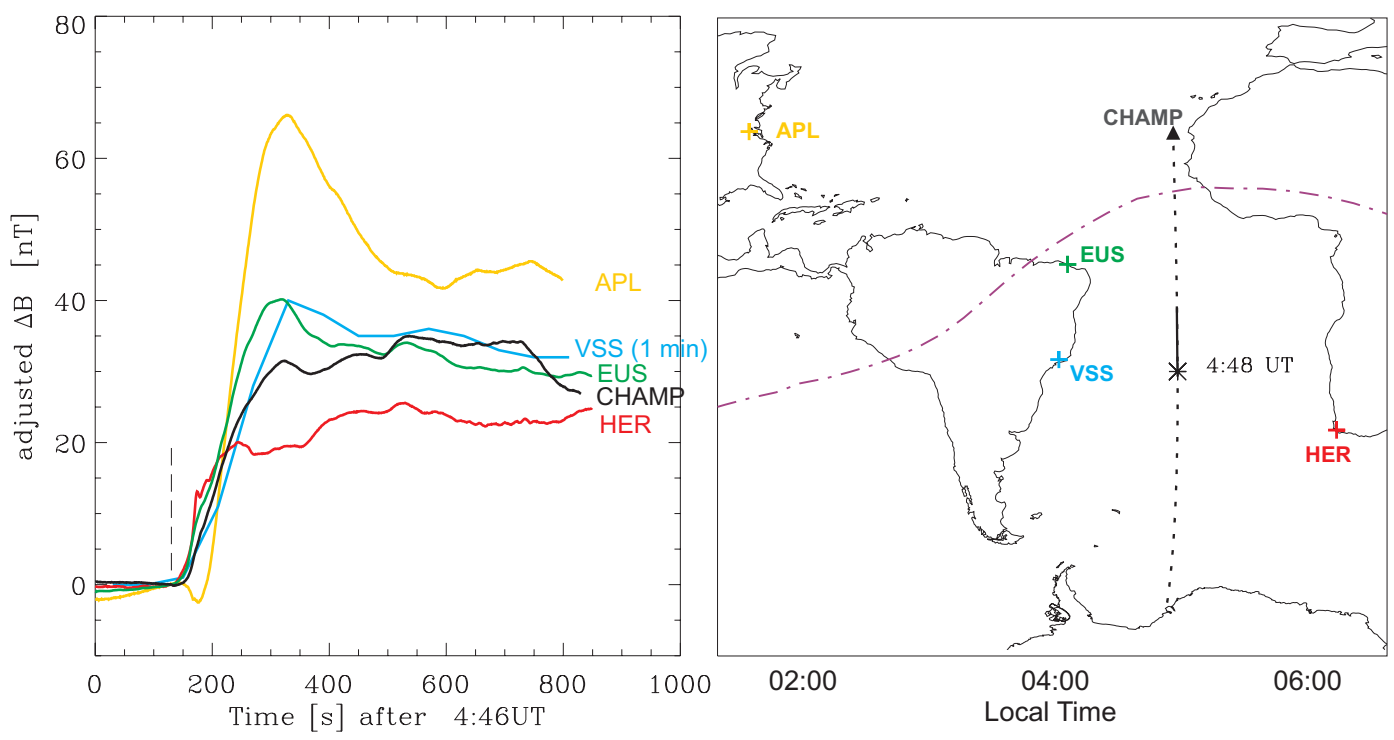

Fig. 4. Same as Fig. 2, but for the SC event on 23 April 2002, when CHAMP flew over the Atlantic.

\section{April 2004}
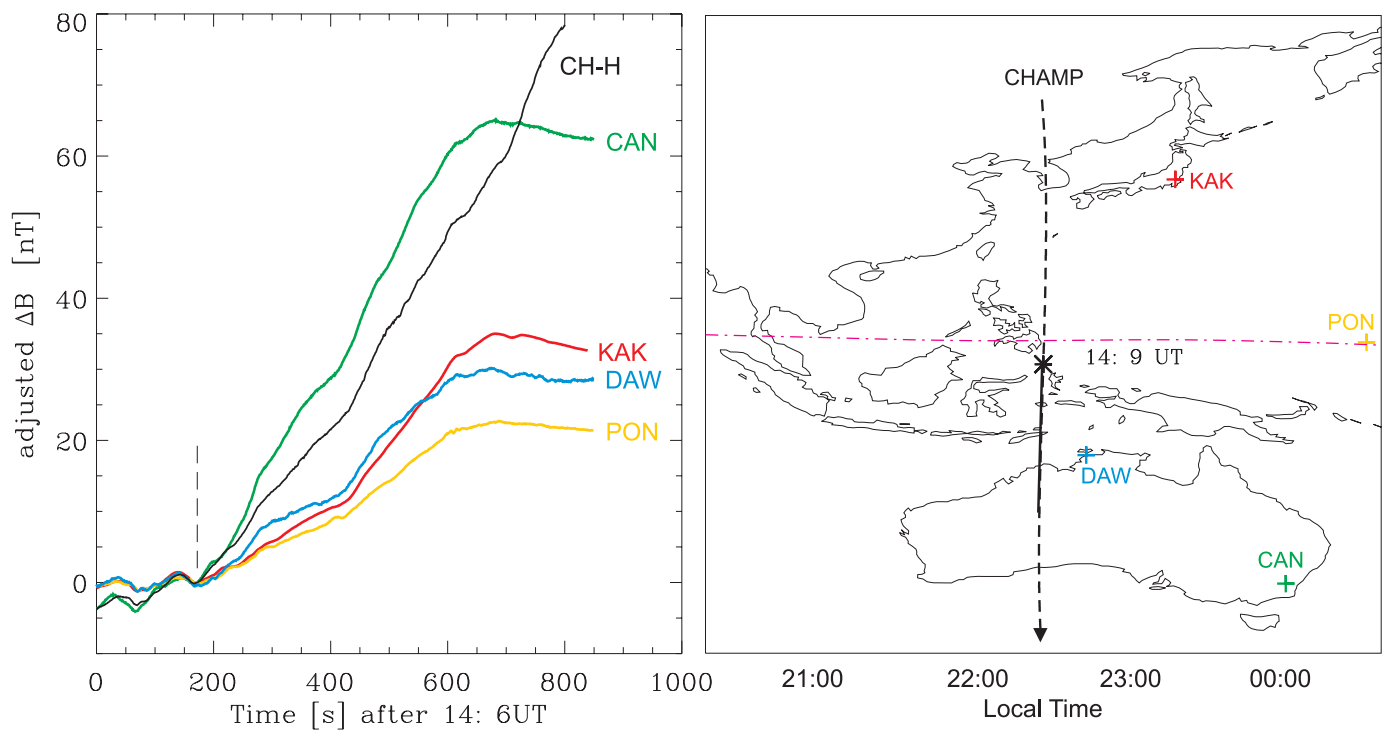

Fig. 5. Same as Fig. 2, but for the SC event on 3 April 2004, when CHAMP flew over Australia.

- After the onset the slope of the five different curves is very similar. This is a confirmation of our assumption that at night a modification of the SC signature by ionospheric currents does not occur, at least not at low latitudes (within $\pm 40^{\circ}$ MLat).

- The magnetic signature of an SC is larger at higher latitudes than in the vicinity of the equator. Examples are observations at APL $\left(50^{\circ}\right)$ and CAN $\left(-43^{\circ}\right)$. Also CHAMP recorded increasing amplitudes towards higher latitude (cf. Fig. 5). This increase at both alti- tude levels implies that ionospheric currents cannot be responsible.

- In some cases there are locally confined differences between the CHAMP observations and near-by ground station recordings. These can generally be explained by currents flowing in regions of ionospheric plasma irregularities. Effects of such currents are not visible on the ground. 


\section{Induction and current effects}

The primary effect of the electric currents induced in the Earth is to reduce or cancel the variation of the radial magnetic field component. From our CHAMP observations we can deduce there is hardly any $\mathrm{Z}$ component variation observed during an $\mathrm{SC}$ event over a significant latitudinal range (cf. Fig. 1). This can mean that the magnetic front approaching the Earth has no radial component and therefore no currents are induced in the ground. Alternatively, if the front has a shape different from the curvature of the Earth; then the induction currents cancel the vertical component. This implies that the resulting SC response is confined to magnetic variations aligned with the Earth surface. This latter scenario is more convincing and seems to be the reason for the latitudinal independence of the $H$ signal recorded by CHAMP. At least, we cannot report any significant differences between the induction effect on ground and at CHAMP altitude.

Another result confirms this conclusion: We investigated the dependence of the field increase rate, $\Delta B / \Delta t$ (cf. column 11 of Table 1), on the amplitude $\Delta B$ of the SCs. We found a weak positive correlation of $\Delta B / \Delta t=30-40 \mathrm{nT} / \mathrm{min}$ for $\Delta B=70-80 \mathrm{nT}$, a dependence very similar to the results of Araki et al. (2004) (their Fig. 2) derived from data of the low latitude station Guam at night.

Our observations confirm the increase of the SC signature at higher latitude (poleward of $40^{\circ}$ MLat). The question is which currents are responsible for it. Ionospheric currents are not a good candidate because we observe larger amplitudes both on the ground and at the satellite. An alternative explanation could be the far-field effect of SC-related field-aligned currents flowing into the auroral region. In order to verify this conjecture we have checked the CHAMP recordings up to auroral latitudes for some suitable events. At magnetic latitudes past $60^{\circ}$ we commonly encounter a westward electrojet flanked by upward and downward FACs on the equatorward and poleward sides, respectively. In addition, we may assume major downward and upward FACs on the dawn and dusk sides feeding the SC-related electrojet. A schematic drawing of the envisaged current system is shown in Fig. 6. The FACs on the dawn and dusk side will produce on the night side an additional northward magnetic signal both on the ground and at satellite height, which gets stronger when approaching the auroral region. Within the auroral region intense Hall and Pedersen currents driven by the primary FACs strongly dominate the SC signal (e.g. Engebretson et al., 1999). At these latitudes the ionospheric conductivity is sufficiently high even on the night side.

The appearance of a PRI at APL near 02:00 LT is not well understood. We checked the SC at College (65.4 MLat) and Barrow $\left(69.6^{\circ}\right)$ in the late evening sector and found a clear $\mathrm{H}$-component PRI also there. The PRI at APL might therefore be explained by an extension of the ionospheric current responsible for the PRI to the location of APL. It might also be caused by FACs associated with the PI, which produce a

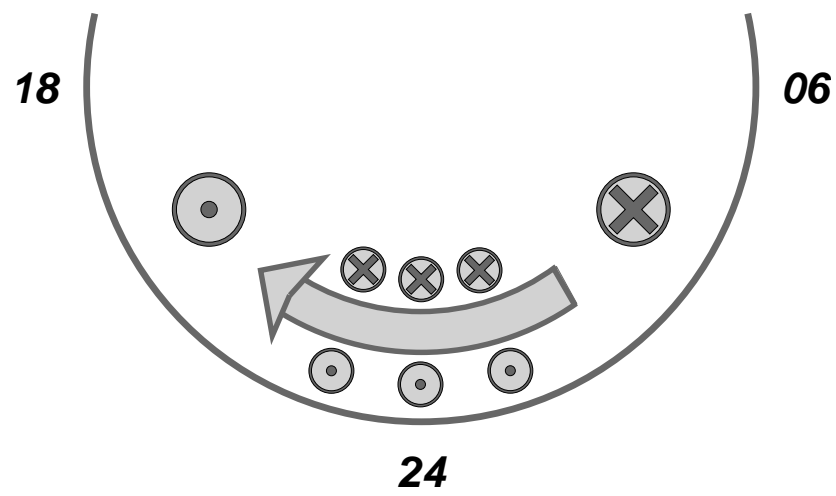

Fig. 6. Schematic drawing of the SC-related auroral current system deduced from our night side magnetic field observations. There seem to be primary FACs, downward at dawn and upward at dusk, that are closed by ionospheric currents. This loop causes poleward pointing magnetic fields at sub-auroral latitudes. North and south of the SC-related current jet we observe FAC sheets.

negative $H$ deflection on the night side (Kikuchi and Araki, 1985). The cause of a PRI will be addressed in more details in the follow-up study focussing on day time SCs.

Although the ionospheric conductivity is low during the night there are some currents flowing in the $\mathrm{F}$ region. These currents are generally connected to regions of enhanced or depleted plasma densities. Relevant candidates are the pressure gradient and the gravity-driven currents (e.g. Lühr et al., 2003; Maus and Lühr, 2006). Neither of them depends on a specific conductivity (like Hall, Pedersen or Cowling). An example of such an SC-unrelated magnetic deflection is shown in Fig. 2 at elapse times past 500 s. Inspection of the CHAMP data revealed that the satellite passed a local plasma feature at that time. In any ground/satellite magnetic field comparison the effect of such local current systems in the F-region have to be taken into account.

\section{Relationship of SCs to solar wind parameters}

For the identification of the solar wind shock causing the SC we used data from the ACE spacecraft. The problem here is that ACE orbits around the Lagrange-1 point, approximately $240 R_{E}$ upstream, whereas we are interested in the corresponding conditions at the magnetopause at around $10 R_{E}$. Therefore, a suitable propagation of the solar wind parameters like velocity, $V$, IMF $B_{z}$, and density, n, has to be applied. We used the method derived by Weimer et al. (2003), with its later modifications by Haaland et al. (2006), for providing the above mentioned parameters at the magnetopause. This method takes into account that IMF and solar wind variations are organised along common surfaces, referred to as "phase fronts", that can be tilted at arbitrary angles with respect to the solar wind velocity. The derived delay times for shifting the ACE data can be considerably differ from the 


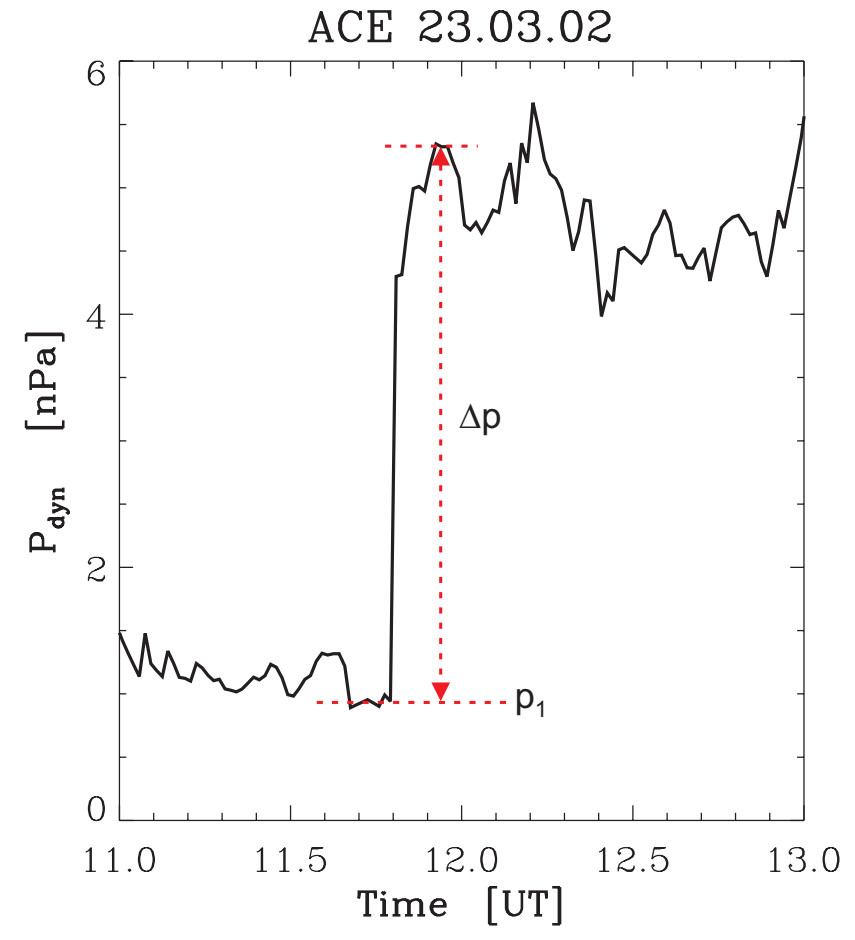

Fig. 7. Typical example of a pressure increase associated with a shock in the solar wind, measured onboard ACE. The time is propagated to the magnetopause (for details see Sect. 2). The quantities $p_{1}$ and $\Delta p$ are used to examine the relationship between the shock input and the caused SC magnetic deflection (listed in Table 1).

standard approach used by many researchers where the delay is determined by just computing the ratio of distance to solar wind speed. Differences between these two methods obtained in propagation time are schematically illustrated in Fig. 2 of Haaland et al. (2007).

The dynamic pressure of the solar wind is calculated as

$p=m n V^{2}$

where $n$ is the solar wind number density, and for $m$ we used 1.15 times the proton mass assuming about $5 \% \alpha$ particles in the solar wind.

Before the arrival of the solar wind shock we assume a balance between the solar wind dynamic pressure and the magnetic pressure at the magnetopause

$p_{1}=\frac{1}{2 \mu_{0}} B_{1}^{2}$

After the shock passage we have

$p_{1}+\Delta p=\frac{1}{2 \mu_{0}}\left(\left(B_{1}+\Delta B\right)^{2}\right)$

and thus with the help of Eq. (3) we obtain the relationship

$\Delta p=\frac{1}{2 \mu_{0}}\left(2 \sqrt{2 \mu_{0} p_{1}} \Delta B+(\Delta B)^{2}\right)$

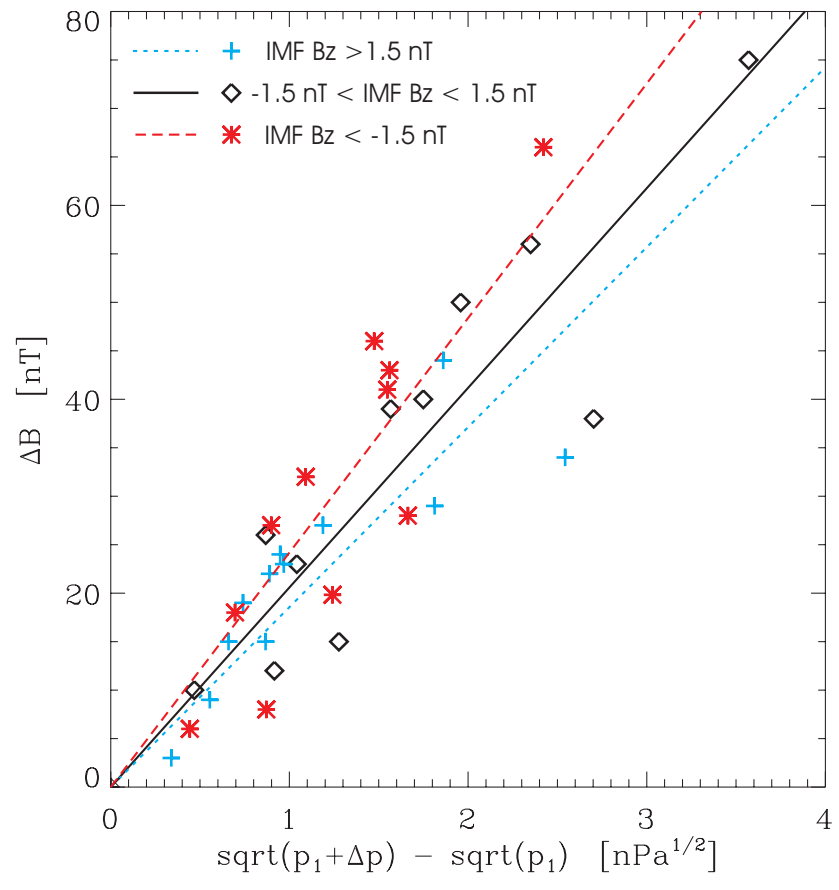

Fig. 8. Amplitude of the SCs (from CHAMP data) as a function of the step in the square root of the solar wind dynamic pressure (from ACE data, see Eq. (7), The events are sorted according to three different ranges of the interplanetary magnetic field, $B_{z}$. The corresponding lines are best fits to the three different data groups.

or

$\Delta B=\sqrt{2 \mu_{0}}\left(\sqrt{\left(p_{1}+\Delta p\right)}-\sqrt{p_{1}}\right)$

Figure 7 shows an example of a typical pressure step in the solar wind. The quantities $p_{1}$ and $\Delta p$ can be easily read from the figure. It should be noted, that the timing of the solar wind data shifted by our propagation method is not precise enough to pinpoint the time difference between the shock arrival and the SC onset. For a meaningful determination of the SC propagation speed from the magnetopause to the satellite location a timing accuracy of the order of $10 \mathrm{~s}$ is required.

In most of the previous studies relating $\Delta B$ with $\Delta p$ (e.g. Siscoe et al., 1968; Araki et al., 1993; Fowler and Russell, 2001) Eq. (6) was used in the form

$\Delta B=\kappa\left(\sqrt{p_{1}+\Delta p}-\sqrt{p_{1}}\right)$

In order to allow a comparison with earlier results we plotted this dependence in Fig. 8. Here $\Delta p$ and $p_{1}$ were obtained from the ACE data as described above and $\triangle B$ was taken from the CHAMP records. Since some indications have been reported that there is a difference in the relationship (7) for positive and negative interplanetary magnetic field (IMF) $B_{z}$ values (e.g. Araki et al., 2006), we separated our dataset in three subsets: clearly positive values $\left(B_{z}>1.5 \mathrm{nT}\right)$, values around zero $\left(-1.5<B_{z}<1.5 \mathrm{nT}\right)$, and clearly negative values $\left(B_{z}<-1.5 \mathrm{nT}\right)$. (According to Smith et al., 1998, the 
one-digit accuracy is relevant since the uncertainty of $B_{z}$ is only $0.1 \mathrm{nT}$ ). Figure 8 indicates at a first glance that the three clouds of points are not strongly separated; nevertheless a $B_{z}$-dependence can be inferred from the three best-fit lines included in the figure. We obtain for the proportionality constant in Eq. (7) values of $\kappa=18.6 \pm 0.2 \mathrm{nT} \mathrm{nPa}^{-1 / 2}$ $\left(B_{z}>1.5 \mathrm{nT}\right), \kappa=20.6 \pm 0.2 \mathrm{nT} \mathrm{nPa}^{-1 / 2} \quad\left(-1.5<B_{z}<1.5 \mathrm{nT}\right)$, and $\quad \kappa=24.7 \pm 0.2 \mathrm{nT} \mathrm{nPa}^{-1 / 2} \quad\left(B_{z}<-1.5 \mathrm{nT}\right)$. Araki et al. (1993) estimated $\kappa$ for night-time cases and obtained values between 10.7 and $11.8 \mathrm{nTnPa}^{-1 / 2}$. Since they assumed a factor of 1.5 for induction effects which was not taken into account in our calculation (cf. Eq. 7), their results should be multiplied by 1.5 when compared to ours which yields a reasonable agreement. These authors also added a table of results from nine other studies which report values in the range of 8.0 to $22.6 \mathrm{nTnPa}^{-1 / 2}$, where night-time values are populating the lower end of the range. Russell et al. (1994) obtained $\kappa=12.3 \mathrm{nT} \mathrm{nPa}^{-1 / 2}$ for night-time events with IMF $B_{z}>0$. It should be kept in mind, however, that all these results refer to SCs observations on the ground. It is obvious, that our $\kappa$-values, derived for SCs observed at $350-450 \mathrm{~km}$ altitude, are larger. The fact that we obtain higher values of $\kappa$ for southward $B_{z}$ is in agreement with the findings of Araki et al. (2006).

According to Eq. (6) we may express $\kappa=\delta \sqrt{2 \mu_{0}}, \delta$ being an attenuation factor of the magnetic response to the pressure impact on the magnetopause. Since $\sqrt{2 \mu_{0}}=50.14 \mathrm{nT} \mathrm{nPa}^{-1 / 2}$, our results show values of $\delta$ between 0.37 and 0.48 . This corresponds to a factor of 2.1-2.7 between the required magnetic field change and the variation observed near the Earth which can be expected. In case of a spherical magnetosphere and a homogeneous external pressure increase, the magnetic field would go up by the same amount everywhere. If we assume a half-sphere affected by the pressure change, which is more like the SC effect on the front side of the magnetosphere, the magnetic field change at the centre is only about half of that at the magnetopause. This theoretical consideration is nicely confirmed by our observations of sufficiently strong SC events.

More insight into the attenuation process is obtained when we calculate the ratio $r=\Delta B_{\text {theory }} / \Delta B_{\text {measured }}$ and plot it versus $\Delta B_{\text {measured }}$ (Fig. 9), where $\Delta B_{\text {theory }}$ is the required magnetic field change calculated from the right hand part of Eq. (6) for compensating the solar wind dynamic pressure increase. For strong $\mathrm{SC}$ events (large $\Delta B_{\text {measured }}$ ) this ratio approaches 2, whereas for weak events (small $\Delta B_{\text {measured) it }}$ is considerably greater. This means that the magnetic disturbance carried as a hydromagnetic wave from the magnetopause towards the Earth is more strongly attenuated for weak SC events than for strong ones. An explanation would be that the impulse transfer from the solar wind to the magnetosphere is more effective for strong SC events than for weak events. We have assumed in our first order pressure balance, Eq. (2), that there is no contribution from the IMF to the solar wind pressure and that the plasma inside the magnetosphere

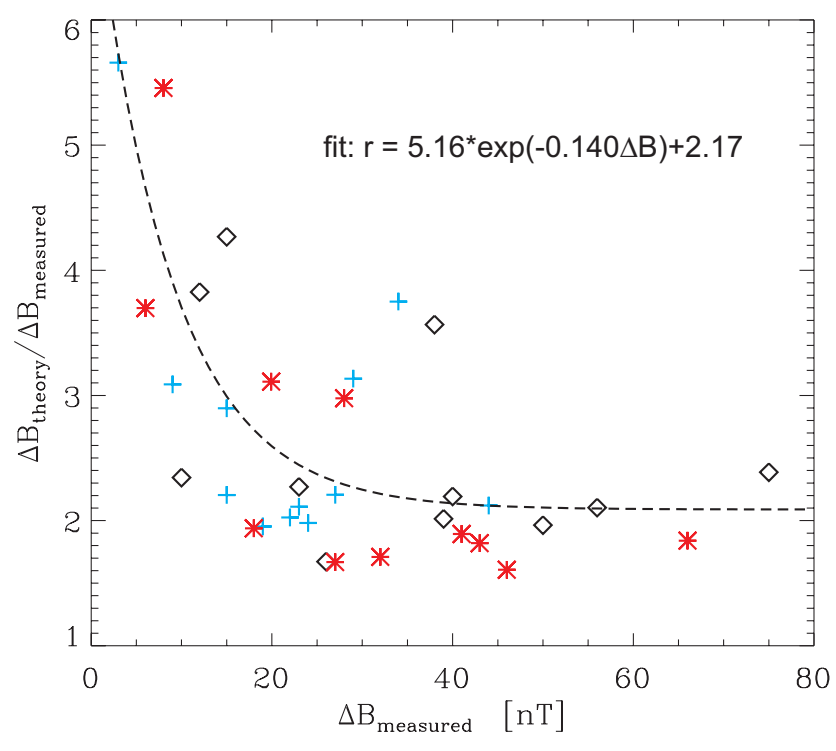

Fig. 9. Plot of the ratio between the expected and observed magnetic field change as a function of $\Delta B_{\text {measured. The expected field }}$ change, $\Delta B_{\text {theory }}$ is calculated from Eq. (6). The different colours correspond to a different orientation of the interplanetary magnetic field. Tentatively an exponential curve of the form $r=a e^{-b \Delta B}+c$ is fitted to the data $(a=5.16, b=0.140, c=2.1)$.

does make no contribution. Both these partial pressures enter Eq. (5) as additive terms. They can only be neglected if the pressure change is large enough. From Fig. 9 we read that this is a reasonable assumption for SCs with amplitudes of more than $20 \mathrm{nT}$ on the night side.

It is obvious that there is considerable scatter in both Figs. 8 and 9. This probably means that there are other factors modifying the impulse transfer from the solar wind to the magnetosphere not accounted for in our approach (Eqs. 26). In more rigorous descriptions of the solar wind dynamic pressure there is a factor $\cos ^{2} \psi$ applied to the right side of Eq. (2), where $\psi$ denotes the angle between the solar wind velocity vector and the normal of the magnetopause at the stagnation point. Such an angle can in principle be derived from the ACE data (Haaland et al., 2007), but it is not very likely that this angle remains constant for the one hour the shock needs to travel from the ACE position to the magnetopause. We tried to apply the $\psi$-dependence, but unfortunately, this increased the scatter of our data points in Figs. 8 and 9 considerably.

\section{Accuracy considerations}

A considerable part of the scatter in our Figs. 8 and 9 is probably just caused by the limited accuracy and reliability of the derived quantities $\Delta B, \Delta p, p_{1}$. Whereas the error in the magnetic field data from CHAMP is quoted to be below $1 \mathrm{nT}$ due to the in-flight calibration with the OVM, larger errors are 
introduced into $\Delta B$ by uncertainties of the POMME-3 model. Its validity is particularly reduced at the fringes of the time interval considered for our study. Here we may find gradients in the baseline of up to $1.5 \mathrm{nT}$ per degree latitude. Other sources of disturbances at satellite level are local current systems associated often with plasma irregularities. They have typical amplitudes of $5 \mathrm{nT}$.

Regarding the solar wind data the greatest uncertainties stem certainly from the propagation to the magnetopause. This holds for the exact timing of the arrival, which we estimate to be uncertain up to $\pm 10 \mathrm{~min}$ and for the precise shape of the shock fronts. Our assumption of an infinite and plane "phase fronts" might be violated, in particular, for a fast moving shock front. A change in orientation of the phase fronts between the ACE position and the magnetopause may, however, alter the impact conditions, with it the compression of the magnetosphere and thus the response of the observed SC. The accuracy of the solar wind parameters themselves is determined by the measurement precision of the instruments onboard ACE (Smith et al., 1998; McComas et al., 1998). The uncertainty introduced by the contribution of $\alpha$ particles to the solar wind pressure, set constant in our analysis, is only of the order of a few percent.

\section{Summary and conclusions}

We have presented a comprehensive study of sudden commencements focussing on low-Earth orbit satellite observations. Our results confirm the assumption that night-time SC signatures are not or only minimally affected by ionospheric currents. This is verified by the comparison of measurements at CHAMP altitude with ground-based observations at magnetic latitudes below about $\pm 40^{\circ}$ (depending on the geomagnetic activity). Neither did we find any significant difference between the onset time of an SC on the ground and at the satellite position, nor differences in the SC amplitude, $\Delta B$ and its increase rate, $\Delta B / \Delta t$, recorded by CHAMP and on the ground that can be related to the Earth induction effects. For the first time it has been shown that the induction effect cancels the vertical component variation such that the $\mathrm{SC}$ magnetic signature is confined to the horizontal component with in the latitude range of $\pm 50^{\circ}$ MLat both on ground and at satellite altitude. A consequence of that is a vanishing induction effect at low latitudes where the SC-related wave front has no vertical component.

The increase of the SC amplitude with latitude is confirmed by our results, but only for magnetic latitudes poleward of about $\pm 40^{\circ}$. As a new result, we attribute this increase in $\mathrm{H}$ component to the far-field effect of field-aligned currents rather than to currents flowing in the ionosphere.

Ionospheric currents originating from locally enhanced or depleted electron density in the F-region are responsible for smaller-scale differences in the magnetic signatures observed by CHAMP and on the ground. These should not be confused with SC effects.

Regarding the relationship between the observed SC amplitude at CHAMP altitudes and the solar wind dynamic pressure changes, we found at low latitudes ( $\pm 40^{\circ}$ MLat) a larger proportionality factor, $\kappa$, for the usually applied relation (7) than earlier SC studies based on ground observations. This is mainly due to an assumed amplification factor of 1.5 from induction effects in the earlier studies. Our larger factor $\kappa$ fits, however, better the expected magnetic field change, supporting our observation that the induction effect is small at low latitudes.

The value of $\kappa$ obtained for specific events is different for weak and strong SCs and it depends additionally on the magnetic latitude, at which it is observed. The obtained ratio between the observed $\kappa$ value and its theoretical expectation, the factor of 0.5 , can be explained by the geometrical shape of the magnetopause.

Our study confirmed a slight difference in the $\kappa$ value for cases occurring during northward or southward IMF, as stated in other studies. We explain the larger $\kappa$ for negative IMF $B_{z}$ by the slightly smaller subsolar distance of the magnetopause for this IMF orientation. A new finding is that for weak SC events (with amplitudes $<20 \mathrm{nT}$ ) the $\Delta B$ changes are significantly smaller than expected from the size of the dynamic pressure step. We regard this as an indication that additional effects such as the solar wind magnetic field and the magnetospheric plasma pressure can no longer be neglected in the momentum balance at the magnetopause.

Acknowledgements. We thank K. Yumoto and A. Ikeda, Space and Earth Electromagnetism Laboratory, Kyushu University, for providing the data from the CPMN, and Mark Moldwin, Institute of Geophysics and Planetary Physics, UCLA for providing help in accessing the MAESURE data. The operational support of the CHAMP mission by the German Aerospace Center (DLR) and the financial support for the data processing by the Federal Ministry of Education and Research (BMBF) are gratefully acknowledged.

Topical Editor M. Pinnock thanks S. Petrinec and another anonymous referee for their help in evaluating this paper.

\section{References}

Araki, T.: Global structure of geomagnetic sudden commencements, Planet. Space Sci., 25, 373-384, 1977.

Araki, T.: A Physical Model of the Geomagnetic Sudden Commencement, in: Solar Wind Sources of Magnetospheric UltraLow-Frequency Waves, edited by: Engebretson, M. J., Takahashi, K., and Scholer, M., AGU Geophys. Monograph 81, p. 183, Washington, D.C., 1994.

Araki, T., Iyemori, T., and Kamei, T.: Sudden commencements observed by MAGSAT above the Ionophere, J. Geomagn. Geoelectr., 36, 507-520, 1984.

Araki, T., Funato, K., Iguchi, T., and Kamei, T.: Direct detection of solar wind dynamic pressure effect on ground geomagnetic field, Geophys. Res. Lett., 20, 775-778, 1993. 
Araki, T., Takeuchi, T., and Araki, Y.: Rise time of geomagnetic sudden commencements - Statistical analysis of ground geomagnetic data, Earth Planet. Space, 56, 289-293, 2004.

Araki, T., Keika, K., Kamei, T., Yang, H., and Alex, S.: Nighttime enhancement of the amplitude of geomagnetic sudden commencements and its dependence on IMF-Bz, Earth Planets Space, 58, 45-50, 2006.

Cahill, L. J., Waite, N. G., Engebretson, M. J., and Sugiura, M.: Toroidal standing waves excited by a storm sudden commencement: DE1 observations, J. Geophys. Res., 95, 7857-7867, 1990.

Engebretson, M. J., Murr, D. L., Hughes, W. J., Lühr, H., Moretto, T., Posch, J. L., Weatherwax, A. T., Rosenberg, T. J., Maclennan, C. G., Lanzerotti, L. J., Marcucci, F., Dennis, S., Burns, G., Bitterly, J., and Bitterly, M.: A multipoint determination of the propagation velocity of a sudden commencement across the polar ionosphere, J. Geophys. Res., 104, 22433-22451, 1999.

Fowler, G. J. and Russell, C. T.: Geomagnetic field response along the polar orbit to rapid changes in the solar wind dynamic pressure, J. Geophys. Res., 106, 18943-18956, 2001.

Haaland, S., Paschmann, G., and Sonnerup, B. U. Ö.: Comment on "A new interpretation of Weimer et al.'s solar wind propagation delay technique" by Bargatze et al., J. Geophys. Res., 111, A06102, doi:10.1029JA011376, 2006.

Haaland, S. E., Paschmann, G., Förster, M., Quinn, J. M., Torbert, R. B., McIlwain, C. E., Vaith, H., Puhl-Quinn, P. A., and Kletzing, C. A.: High-latitude plasma convection from Cluster EDI measurements: method and IMF-dependence, Ann. Geophys., 25, 239-253, 2007, http://www.ann-geophys.net/25/239/2007/.

Han, De-Sheng, Araki, T., Yang, H.-G., Chen, Z.-T., Iyemori, T., and Stauning, P.: Comparative study of geomagnetic sudden commencements (SC) between Oersted and ground observations at different local times, J. Geophys. Res., 112, A05226, doi:10.1029/2006JA011953, 2007.

Kikuchi, T. and Araki, T.: Preliminary positive impulse of geomagnetic sudden commencement observed at dayside middle and low latitudes, J. Geophys. Res., 90, 12195-12200, 1985.

Kikuchi, T., Tsunomura, S., Hashimoto, K., and Nozaki, K.: Fieldaligned current effects on middle latitude geomagnetic sudden commencements, J. Geophys. Res., 106, 15555-15565, 2001.

Kokubun, S.: Characteristics of storm sudden commencement at geostationary orbit, J. Geophys. Res., 88, 10025-10033, 1983.

Lühr, H., Rother, M., Maus, S., Mai, W., and Cooke, D.: The diamagnetic effect of the equatorial Appleton anomaly: Its characteristics and impact on geomagnetic field modelling, Geophys. Res. Lett., 30(17), 1906, doi:10.1029/2003GL017407, 2003.
Maus, S. and Lühr, H.: A gravity-driven electric current in the Earth's ionosphere identified in CHAMP satellite magnetic measurements, Geophys. Res. Lett., 33, L02812, doi:10.1029/2005GL024436, 2006.

Maus, S., Rother, M., Stolle, C., Mai, W., Choi, S.-C., Lühr, H., Cooke, D., and Roth, C.: Third generation of the Potsdam Magnetic Model of the Earth (POMME), Geochem. Geophys. Geosyst., 7, Q07008, doi:10.1029GC001269, 2006.

McComas, D. J., Bame, S. J., Barker, P., Feldman, W. C., Phillips, J. L., Riley, P., and Griffee, J. W.: Solar Wind Electron Proton Alpha Monitor (SWEPAM) for the Advanced Composition Explorer, Space Sci. Rev., 86, 563-612, 1998.

Patel, V. L.: Sudden impulses in the geomagnetotail and the vicinity, Planet. Space Sci., 20, 1127-1136, 1972.

Petrinec, S. M., Yumoto, K., Lühr, H., Orr, D., Milling, D., Hayashi, K., Kokubun, S., and Araki, T.: The CME event of February 21, 1994: Response of the magnetic field at the Earth's surface, J. Geomag. Geoelectr., 48, 1341-1379, 1996.

Reigber, Ch., Lühr, H., and Schwintzer, P.: CHAMP Mission Status, Adv. Space Res., 30(2), 129-134, 2002.

Russell, C. T., Ginskey, M., and Petrinec, S.: Sudden impulses at low latitude stations: Steady state response for northward interplanetary magnetic field, J. Geophys. Res., 99, 253-261, 1994.

Shinbori, A., Ono, T., Iizima, M., and Kumamoto, A.: SC related electric and magnetic field phenomena observed by the Akebono satellite inside the plasmasphere, Earth Planets Space, 56, 269282, 2004.

Siscoe, G. L., Formisano, V., and Lazarus, A. J.: Relation between geomagnetic sudden impulse and solar wind pressure changes An experimental investigation, J. Geophys. Res., 73, 4869-4874, 1968.

Smith, C. W., L’Heureux, J., Ness, N. F., Acuña, M. H., Burlaga, L. F., and Scheifele, J.: The ACE Magnetic Field Experiment, Space Sci. Rev., 86, 613-632, 1998.

Weimer, D. R., Ober, D. M., Maynard, N. C., Collier, M. R., McCormas, D. J., Ness, N. F., Smith, C. W., and Watermann, J.: Predicting interplanetary magnetic field (IMF) propagation delay times using the minimum variance technique, J. Geophys. Res., 108(A1), 1026, doi:10.1029/2002JA009405, 2003.

Wilken, B., Goertz, C. K., Baker, D. N., Higbie, P. R., and Fritz, T. A.: The SSC on July 29, 1977 and its propagation within the magnetosphere, J. Geophys. Res., 87, 5901-5910, 1982. 